\title{
Hybrid Convolutional Neural Network with PSO Based Severe Dengue Prognosis Method in Human Genome Data
}

\author{
${ }^{1}$ Mohammed Mustafa, ${ }^{2}$ Rihab Eltayeb Ahmed, \& ${ }^{3 S a r a h ~ M u s t a f a ~ E l j a c k ~}$ \\ ${ }^{1}$ Department of Computer Science, University of Tabuk, Tabuk City, Saudi Arabia. \\ ${ }^{2}$ Faculty of Computers and information Technology, University of Tabuk, Tabuk City, Saudi Arabia. \\ 3Majmaah University, Department of Computer Science and Information College of Science Al Zulfi, Saudi Arabia. \\ ${ }^{* *}$ Corresponding Author: mmustafa@ut.edu.sa
}

Received: 05.01.2020, Revised: 08.02.2020, Accepted: 17.03.2020, Published: 30.03 .2020

DOI:

10.53409/mnaa.jcsit1104

Abstract: Dengue is one of the most significant diseases transmitted by arthropods in the world. Dengue phenotypes are focused on documented inaccuracies in the laboratory and clinical studies. In countries with a high incidence of this disease, early diagnosis of dengue is still a concern for public health. Deep learning has been developed as a highly versatile and accurate methodology for classification and regression, which requires small adjustment, interpretable results, and the prediction of risk for complex diseases. This work is motivated by the inclusion of the Particle Swarm Optimization (PSO) algorithm for the fine-tuning of the model's parameters in the convolutional neural network (CNN). The use of this PSO was used to forecast patients with extreme dengue, and to refine the input weight vector and $\mathrm{CNN}$ parameters to achieve anticipated precision, and to prevent premature convergence towards local optimum conditions.

Keywords: Deep learning, Particle Swarm Optimization, convolutional neural network and Dengue.

\section{INTRODUCTION}

ntil September this year, dengue has U claimed the lives of more than 80 people and affected about 40,000 people across the world. According to statistics under the Ministry of Health's National Vector Borne Disease Control Program (NVBDCP), 83 people have died until 30 September, while 40,868 have been affected. Last year, 325 people were killed, and some 1, 88,401 people were affected by the mosquitoborne tropical disease. Dengue had claimed 35 lives in Kerala and 3,660 had been affected by the State until 30 September 2018. In Maharashtra, 18 people have died and 4,667 have suffered from the disease, according to data [1].

Early forecast factors and algorithms for moderate or severe Dengue prediction were investigated in previous studies. Nevertheless, it is difficult to draw specific conclusions in the case populations (adults vs. infants, hospitalized vs. outpatients) or clinical concepts of "serious" dengue [2] [3]. However, the positive and negative forecast values of the prognostic tool were not reported in previous prognostic studies [4]. In that sense, a practical, forecasting method using CNN-PSO to allow the early prognosis for severe dengue was developed in this current study.

This is the structure of the remaining paper. Section 2 addresses the related plays. The proposed methods of describing the detection of severe dengue using CNN-PSO are defined in Section 3. The experimental findings obtained in Section 4 are discussed. The conclusion and future work are contained in Section 5 .

\section{RELATED WORK}

Specific methods have been suggested in the literature to identify lung nodules using CT dengue. A non-invasive precise diagnostic device was built in [5], which could allow doctors to assess the rates of risk of dengue patients and thus make the right choice. The adaptive neurofuzzy inference system (ANFIS) was used to construct the diagnostic model with observations for bioelectronic impedance analysis, with the symptoms or signs provided to dengue patients.

This study [6] aimed to develop a noninvasive diagnostic system to help doctors 
classify the risk of dengue-related people using the Levenberg / Marquardt and Scaled Conjugate Gradient algorithms Multilayer Perceptron Neural Network (MPNN) models.

The new Arbovirus Dengue Diagnosis Fusion Architecture was introduced in [7]. The software incorporates characteristics of platelets and CBR to promote the evaluation of the health system. In [8], the early diagnosis of dengue fever was based on a hybrid Artificial Intelligence System, Adaptive Neuro-Fuzzy Inference System (ANFIS). Early dengue disease clinical signs are unspecific and overlap the other infectious diseases.

In [9], attempts have been made to establish an earlier diagnostic model of dengue fever based on PSO-ANN. The PSO methodology used in the proposed model is to optimize ANN process weight and biais parameters. In [10] proposed a new artificial intelligence-based methodology which predicts diagnostics in real time, by using an alternative decision tree approach that promotes highly accurate rules for the generation of false-positive and falsenegative diagnosis.

\section{PROPOSED METHODOLOGY}

The CNN-PSO is theoretical method is used to predict genome data for dengue. The features are entered as CNN-PSO input following pre-processing of the sample dataset and further prediction of severe dengue is done effectively. Figure 1. Illustrates the architectural diagram for the proposed CNN-PSO dengue forecast.

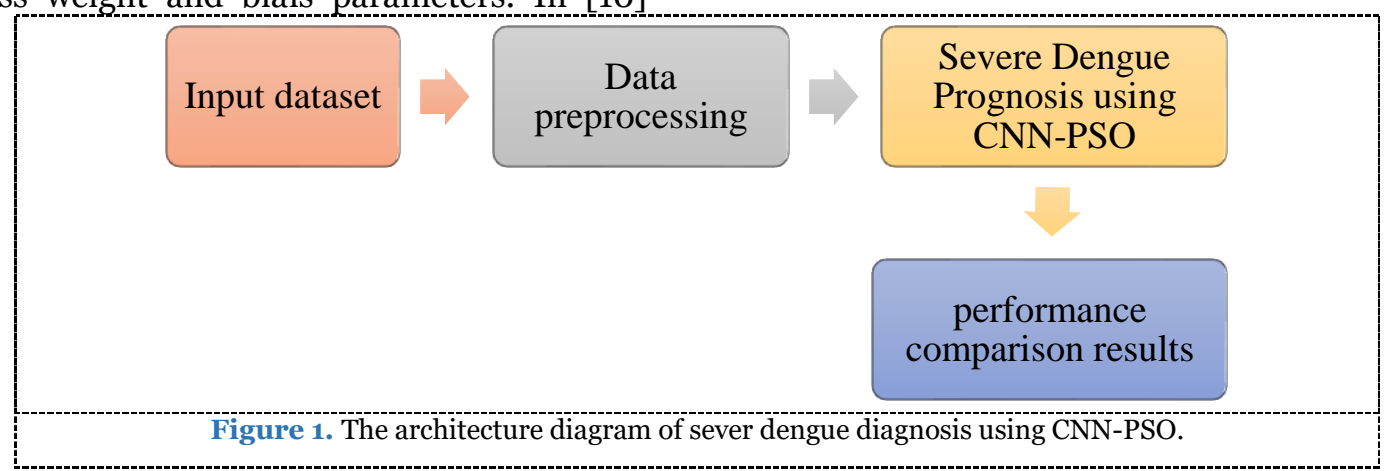

\subsection{Patient Cohort and Preprocessing of data}

The three hospitals of Recife, Brazil have been screened and invited to participate in this study for patients with dengue-related symptoms. After a detailed explanation of the study proposed, all patients who decided to participate were included in this research. FIOCRUZPE: CEP / CPQAM No11/11 and C.A.A.E. 0009.0.095.000-11, IORGooo1419 reviewed and accepted the report. Whole blood was collected and tested for hematocrit and hemogram, white blood cell count, differential leukocyte count, serum albumin and serum aspartate (AST), and serum alanin transaminase (ALT) performed by standardized analytics from enrolled patients. The patient data is summarized in the patient cohort.

Data Pre-processing: The genome data is composed of 102 genotypes determined at 322 polymorphisms of the loci. The data were encrypted into indicators using a categorical schema to establish an SNP-genotype attribute that is homozygous dominant, heterozygous, or homozygous recessive. The lack of information at a forum was treated as a further category.

\subsection{Dengue diagnosis Using CNN-PSO}

In the original data set, $75 \%$ sample points were used to train the CNN, while the other $25 \%$ samples have been reserved for the test set. This study proposes transferring learning based on pretrained deep learning. A number of parameters must be determined before the training stage in the $\mathrm{CNN}$ [11] algorithms. These parameters may influence the classification results more or less depending on the application. After the random experiments, one should consider which parameters have more effect. In order to determine the best value, one could be statistically evaluated as a factor in the whole factorial test. The algorithm defaulted to set other parameters with less impact on the results.

Convolutional Neural Network: CNN is an approach to machine learning focused on the profound structure of the brain [11]. This network is essentially comprised of three layers: concentration, sub-sampling or pooling and full link layers. So each layer is briefly described in the following sections.

Convolution Layer: Convolution Layer: There are a number of $n$ filters in each layer. The number of layers used for the convolution procedure is the 
input of those filters and the depth of the feature(s) generated is proportional to the number of filters. Recognize that another filter is perceived to be a special feature in a certain data set attribute.

The output of the $l$-th convolution layer, represented as $\operatorname{Conv}_{j}^{(l)}$, encompasses of feature attributes. It is computed as

$$
\operatorname{Conv}_{i}^{(l)}=\operatorname{bias}_{i}^{(l)}+\sum_{j=1}^{a_{i}^{(l-1)}} K_{i, j}^{(l-1)} * C_{j}^{(l)}
$$

Where bias $_{i}^{(l)}$ stands the bias matrix and $K_{i, j}^{(l-1)}$ stands convolution filter or kernel of size $a * a$ that connects the $\mathrm{j}$-th feature in layer $(l-1)$ through $i$-th feature in the same layer also the output layer contains of feature. The input space in (1) is the primary convolutional layer, $\operatorname{Conv}_{i}^{(0)}=$ $X_{i}$

The functionality is given by the kernel. Subsequently the convolution layer, the activation function will trigger the nonlinear transformation of the output of the convolutional layer:

$$
Y_{i}^{(l)}=Y\left(\operatorname{Conv}_{i}^{(l)}\right)
$$

where $Y_{i}^{(l)}$ remains the output of the activation function besides $\operatorname{Conv}_{i}^{(l)}$ remains the input that it receives.

Normally used activation functions are sigmoid, tanh, and rectified linear units (ReLUs). In this work, ReLUs which is signified as $Y_{i}^{(l)}=$ $\max \left(0, Y_{i}^{(l)}\right)$ are used.

Fully

Connected

layer:

The end layer of the $\mathrm{CNN}$ is a typical

network of reviews with one or more hidden colum ns. Softmax activation function is used in the output layer:

$$
y_{i}^{(l)}=\sum_{i=1}^{m_{i}^{(l-1)}} w_{i}^{(l-1)} y_{i}^{(l-1)}
$$

where $w_{i}^{(l-1)}$ remains the weight to be modified by the completely connected sheet, the transfer function represents nonlinearity to form the representation of each class. Note that nonlinearity of the fully connected layer is not contained in its neurons, not in separate layers, as in convolutions and pooling layers. While examining for feedback signals, the development of the $\mathrm{CNN}$ is begun. Training takes place using the stochastic gradient descent method [11]. Then use a single example from a workout, the algorithm explores the gradients. Following planning, the parameters of $\mathrm{CNN}$ are calculated.

\subsection{Hyper parameter tuning of CNN using PSO}

The neurons, weight and bias parameters to be optimized by PSO and parameter setting of PSO are shown in Table 1.

Table 1. Parameter setting of PSO

\begin{tabular}{cc}
\hline Parameter & values \\
\hline Population of PSO $(\mathrm{p})$ & 15 \\
\hline PSO stop criteria & 50 \\
\hline Velocity coefficient $\left(\boldsymbol{v}_{\boldsymbol{c} 1}, \boldsymbol{v}_{c 2}\right)$ & 2 \\
\hline Hindering coefficient $(\boldsymbol{w})$ & 15 \\
\hline $\begin{array}{c}\text { Max and Min value of Hindering } \\
\text { coefficient }\left(\boldsymbol{w}_{\max } \& \boldsymbol{w}_{\min }\right)\end{array}$ & $\begin{array}{c}0.9 \& \text { o.4 } \\
\text { respectively }\end{array}$ \\
\hline Number of particle dimension $(\boldsymbol{d})$ & 8 \\
\hline
\end{tabular}

PSO produces and evolves several solutions to a single problem over many centuries. Each solution contains all parameters to improve the results. Weights in both layers contribute to CNN's high precision. A single PSO solution would therefore be usable in all $\mathrm{CNN}$ weights. The $\mathrm{CNN}$ has four levels ( 1 input, 2 hidden and 1 output), according to the network structure discussed in the previous tutorial and shown in the figure below. Recall that PSO uses a fitness function of precision to assign each solution a fitness value and the higher the fitness value, the better the response. The following steps are given:

Step 1 (Solution Defining Space and Fitness Functions): Pick optimized parameters. Put this in an appropriate range with the optimum solution. Instead, in a multidimensional optimisation, set a minimum and maximum value for each dimension.

Step 2 (Initialization phase): Determine population size and maximum iteration number. Set the starting position for each particle and start speed. Accuracy is used to calculate the error between the prediction value and the true data for every particle.

Step 3 (optimization phase): first, find the best part position and best part position in the swarm in its history. Second, positions and speeds are upgraded by equations (4) to (6) where the weight of inertia and the learning factor are concerned.

$$
x_{k}^{s+1}=x_{k}^{s}+v_{k}^{s+1}
$$




$$
\begin{aligned}
& v_{k}^{s+1} \\
& =w v_{k}^{s}+v_{c 1} \cdot \text { rand } \cdot\left(x_{\text {pbest }(k)}^{s}-x_{k}^{s}\right) \\
& +v_{c 2} \cdot \text { rand } \cdot\left(x_{\text {gbest }(k)}^{s}-x_{k}^{s}\right) \\
& \quad w=w_{\text {max }}-\left(w_{\text {max }}-w_{\text {min }}\right) * \frac{s}{I}
\end{aligned}
$$

In Equations (4) to (6) above, $v_{k}^{t+1}$ and $x_{k}^{t+1}$ are the $s$ is the speed and position components of the $k$ th particle, $v_{c 1}, v_{c 2}$ are the Velocity coefficients and the pbest $(k)$ and $g$ best $(k)$ are personal best and global best particle. Here the $w$ hindering coefficient as it helps the particles to move by hindering towards better positions and finally rand is a uniform random value between 0 and 1 .

Step 4: If the evaluation function (predicted accuracy of the training information) is converging, the optimization will end; otherwise, proceed to step 3 and Step 4 is finished finally.

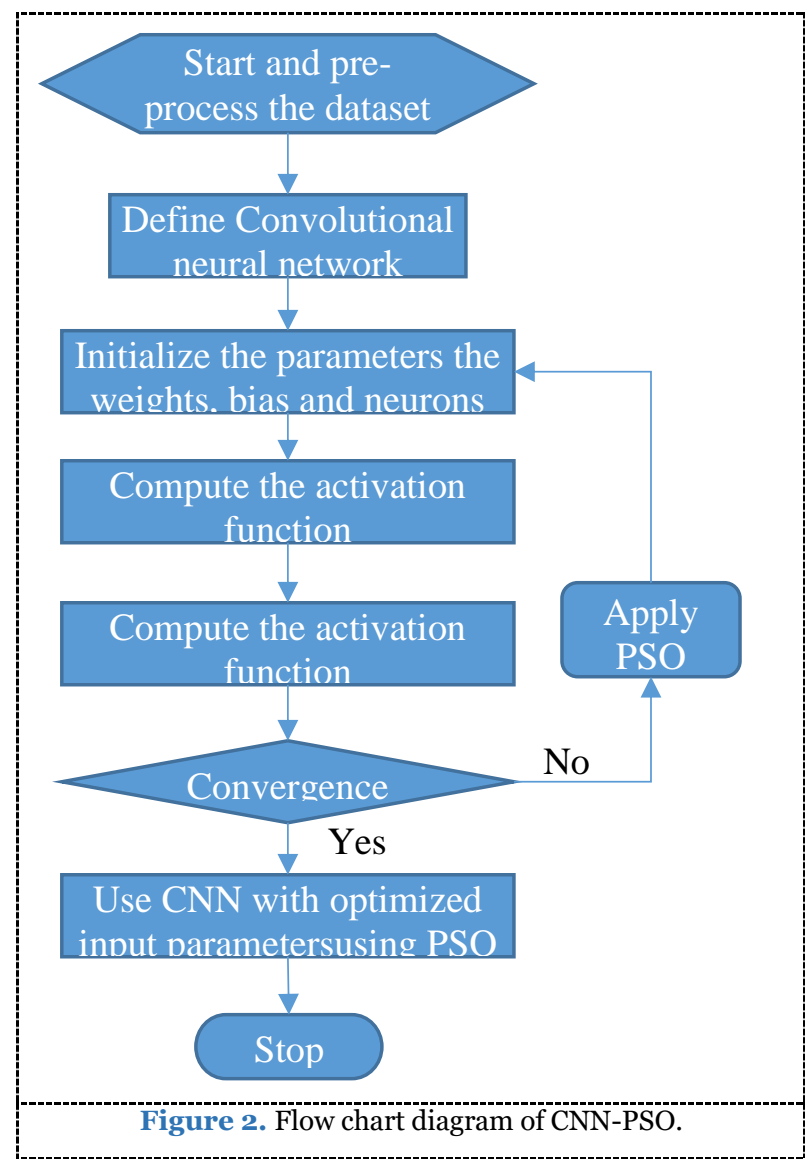

Therefore the description of each particle is such that the one part contains variables (particles) with discrete values (that represent the neuron numbers for each layer). The other part contains variables with continuous values (that represent the weights and bias values). At this stage, this specification is used to create one random initial population. Using particulate values in the determined population, the $\mathrm{CNN}$ architecture and training weights and distortions are specified. PSO trains its fitness function for every chromosome, which is determined for this population, then via the standard input data, heights, weights and biases proposed for this. Figure 2 shows the flow chart of CNN-PSO.

\section{EXPERIMENTAL RESULTS AND DISCUSSION}

The performance of the proposed CNN-PSO is evaluated in this section, and the performance results are compared with existing MPNN [6], ANFIS [8] and PSO-ANN [9] schemes. The performance measurement is done in terms of precision, f-measurement, recall and accuracy.

Precision: It reflects the proportion of positive samples correctly classified as expected in equation (7):

Precision $=\frac{T P}{F P+T P}$

Recall: The recall of a classifier reflects the positive samples properly assigned to the total number of positive samples and is calculated as in equation (8):

Recall $=\frac{T P}{T P+F N}$

F-measure: this is also referred to as F 1-score, and as in equation (9) is the harmonic mean of precision and recall:

$F-$ measure $=\frac{2 *(\text { Recall } * \text { Precision })}{(\text { Recall }+ \text { Precision })}$

Accuracy: This is one of the most frequently used performance classification measures and is defined as a ratio between the correctly classified samples and the total number of samples as in equation (10):

Accuracy $=\frac{T P+T N}{T P+T N+F P+F N}$

Where true positive (TP) samples are properly classified as no dengue, false positive (FP) samples are incorrectly classified as dengue, True negative (TN) samples are properly classified as dengue, and false negatives (FN) are incorrectly classified as dengue.

\subsection{Precision Rate comparison}

From the above Figure 3, the graph shows how accurate the number of images in the specified datasets is compared. These methods are implemented as MPNN, ANFIS, PSO-ANN and CNN-PSO. When the number of records increases according to the precision value. From this graph, it is learned that the proposed CNN-PSO offers $94 \%$ 
higher precision than previous methods that yield better results in the classification of lung nodules due to hyper parameter optimization of CNN using PSO. The numerical results of Precision Comparison is shown in Table 2.

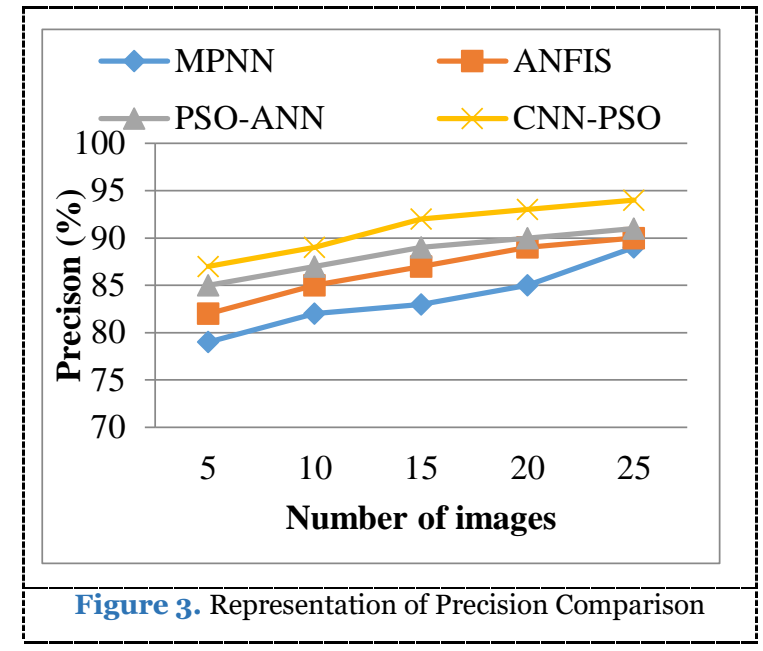

Table 2. The numerical results of Precision Comparison

\begin{tabular}{ccccc}
\hline $\begin{array}{c}\text { No. of } \\
\text { images }\end{array}$ & MPNN & ANFIS & $\begin{array}{c}\text { PSO- } \\
\text { ANN }\end{array}$ & $\begin{array}{c}\text { CNN- } \\
\text { PSO }\end{array}$ \\
\hline 5 & 79 & 82 & 85 & 87 \\
\hline 10 & 82 & 85 & 87 & 89 \\
\hline 15 & 83 & 87 & 89 & 92 \\
\hline 20 & 85 & 89 & 90 & 93 \\
\hline 25 & 89 & 90 & 91 & 94 \\
\hline
\end{tabular}

\subsection{Recall comparison}

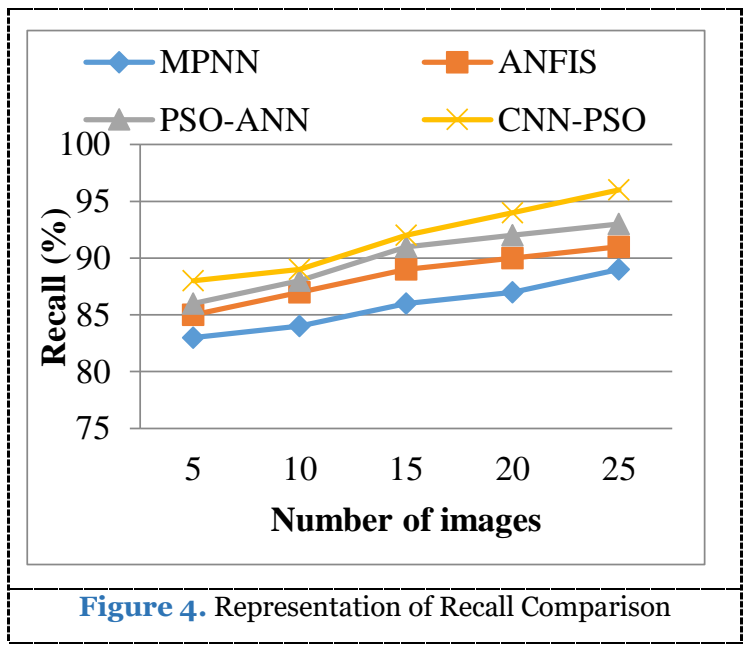

From the above Figure 4, the graph illustrates the recall relation for the number of images in the listed datasets. These methods are implemented as MPNN, ANFIS, PSO-ANN and CNN-PSO. Increasing the number of photographs often increases the correct value for the recall. Through this graph, it is discovered that the current CNNPSO offers recall 96\% higher than previous methods. The explanation for this is that the CNNPSO extracts the features directly which will enhance the detection of severe dengue. The numerical results of Recall Comparison is shown in Table 3 .

Table 3. The numerical results of Recall Comparison

\begin{tabular}{ccccc}
\hline $\begin{array}{c}\text { No. of } \\
\text { images }\end{array}$ & MPNN & ANFIS & $\begin{array}{c}\text { PSO- } \\
\text { ANN }\end{array}$ & $\begin{array}{c}\text { CNN- } \\
\text { PSO }\end{array}$ \\
\hline 5 & 83 & 85 & 86 & 88 \\
\hline 10 & 84 & 87 & 88 & 89 \\
\hline 15 & 86 & 89 & 91 & 92 \\
\hline 20 & 87 & 90 & 92 & 94 \\
\hline 25 & 89 & 91 & 93 & 96 \\
\hline
\end{tabular}

\subsection{F-measure Rate comparison}

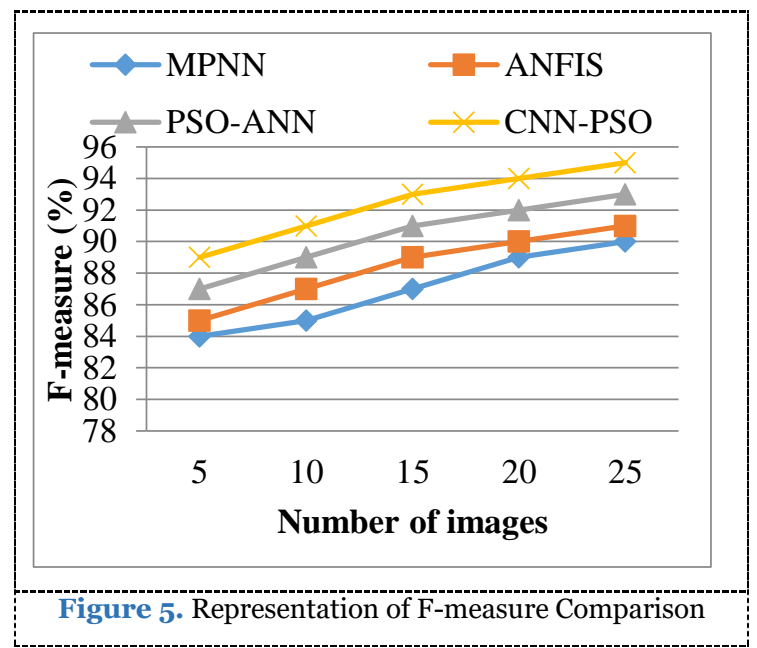

From the above Figure 5, the graph explains the f-measure relation for the number of images in the given datasets. These methods are implemented as MPNN, ANFIS, PSO-ANN and CNN-PSO. When the number of data is increased, and the f-measure value is increased accordingly.

Table 4. The numerical results of F-measure Comparison

\begin{tabular}{ccccc}
\hline $\begin{array}{c}\text { No. of } \\
\text { images }\end{array}$ & MPNN & ANFIS & $\begin{array}{c}\text { PSO- } \\
\text { ANN }\end{array}$ & $\begin{array}{c}\text { CNN- } \\
\text { PSO }\end{array}$ \\
\hline 5 & 84 & 85 & 87 & 89 \\
\hline 10 & 85 & 87 & 89 & 91 \\
\hline 15 & 87 & 89 & 91 & 93 \\
\hline 20 & 89 & 90 & 92 & 94 \\
\hline 25 & 90 & 91 & 93 & 95 \\
\hline
\end{tabular}


From this graph it is learned that the proposed CNN-PSO offers 95\% higher f-measurement than previous methods. Therefore the proposed $\mathrm{CNN}$ PSO algorithm is stronger than the current algorithms in terms of better performance of prognosis of severe dengue. The numerical results of F-measure Comparison is shown in Table 4.

\subsection{Accuracy comparison}

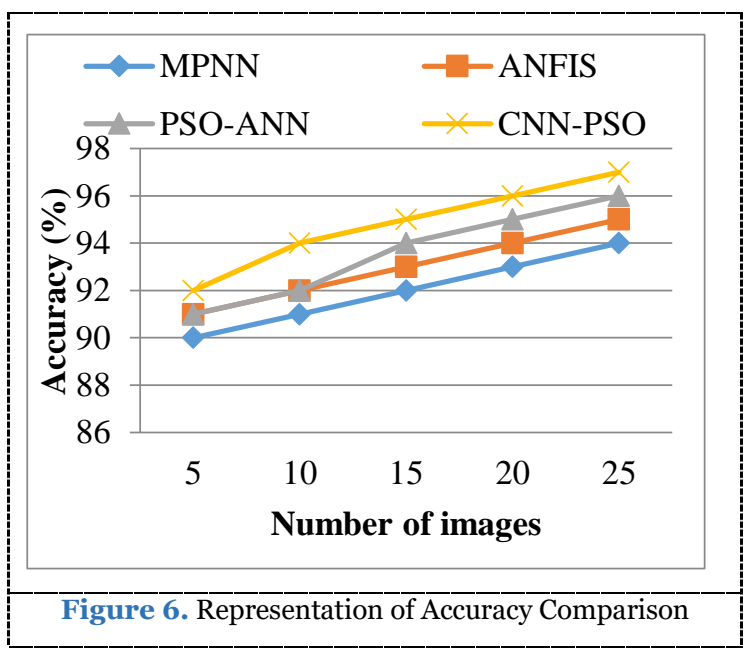

From the above Figure 6, the diagram illustrates the processing time relation for the number of images in the specified datasets. These methods are implemented as MPNN, ANFIS, PSOANN and CNN-PSO. From this graph it is known that the proposed CNN-PSO algorithm is higher than the existing algorithms with a high precision rate of $97 \%$ in terms of better template matching results. This is due to the automatic extraction of the function in the CNN-PSO algorithm, which increases the severe dengue prognosis. The numerical results of Accuracy Comparison is shown in Table 5 .

Table 5. The numerical results of Accuracy Comparison

\begin{tabular}{ccccc}
\hline $\begin{array}{c}\text { No. of } \\
\text { images }\end{array}$ & MPNN & ANFIS & $\begin{array}{c}\text { PSO- } \\
\text { ANN }\end{array}$ & $\begin{array}{c}\text { CNN- } \\
\text { PSO }\end{array}$ \\
\hline 5 & 90 & 91 & 91 & 92 \\
\hline 10 & 91 & 92 & 92 & 94 \\
\hline 15 & 92 & 93 & 94 & 95 \\
\hline 20 & 93 & 94 & 95 & 96 \\
\hline 25 & 94 & 95 & 96 & 97 \\
\hline
\end{tabular}

\section{CONCLUSION AND FUTURE WORK}

In this work, a forecast of severe dengue, based on CNN-PSO, is proposed. The primary advantages of a CNN-based genome method for the prediction of serious dengue development are that it can also be implemented before infection at any stage of disease, and that human samples can be widely selected. The results show that the presented methodology offers a robust tool for pronouncing dengue severity. Experimental data show that the built CNN-PSO can detect most of the nodules with a high $97 \%$ accuracy rate. This success is mainly due to the deep CNN structure, which uses the capacity to extract different level features and to the use of PSO to modify hyperparameters that has resulted in better generalization. Smart techniques along with evolutionary algorithms can be used for faster calculation by choosing the optimisation required in different aspects. Considering multi-target distinguishing changes, the measurement accuracy is a good attempt.

ETHICS APPROVAL AND CONSENT TO PARTICIPATE

Not applicable.

\section{HUMAN AND ANIMAL RIGHTS}

No animals/humans were used for studies that are basis of this research.

\section{CONSENT FOR PUBLICATION}

Not applicable.

\section{AVAILABILITY OF DATA AND MATERIALS}

The authors confirm that the data supporting the findings of this research are available within the article.

\section{FUNDING}

None.

\section{CONFLICT OF INTEREST}

The authors declare no conflict of interest, financial or otherwise.

\section{ACKNOWLEDGEMENTS}

The authors would like to thank their present employer for providing support while carrying out this research work. 


\section{REFERENCES}

[1]. Ta-Chien Chan, Tsuey-Hwa Hu, Jing-Shiang Hwang, "Daily forecast of dengue fever incidents for urban villages in a city", International Journal of Health Geographics, 2015.

[2]. Khan MI Anwar E Agha A et al. Factors predicting severe dengue in patients with dengue fever. Mediterr $\mathrm{J}$ Hematol Infect Dis 2013; 5:e2013014.

[3]. Ho TS Wang SM Lin YS Liu CC . Clinical and laboratory predictive markers for acute dengue infection. $J$ Biomed Sci,2013; 20:75.

[4]. Falconar AK Romero-Vivas CM . Simple prognostic criteria can definitively identify patients who develop severe versus non-severe dengue disease, or have other febrile illnesses. $J$ Clin Med Res, 2012; 4:33-44.

[5]. Faisal T, Taib MN, Ibrahim F. Adaptive Neuro-Fuzzy Inference System for diagnosis risk in dengue patients. Expert Systems with Applications. 2012 Mar 1;39(4):448395 .

[6]. Faisal T, Taib MN, Ibrahim F. Neural network diagnostic system for dengue patients risk classification. Journal of medical systems. 2012 Apr 1;36(2):661-76.

[7]. Jiji GW, Lakshmi VS, Lakshmi KV, Priya SS. Diagnosis and Prognosis of the Arbovirus-Dengue using Intelligent Algorithm. Journal of The Institution of Engineers (India): Series B. 2016 Jun 1;97(2):115-20.

[8]. Saikia D, Dutta JC. Adaptive Network Based Fuzzy Inference System for Early Diagnosis of Dengue Disease. InAdvances in Computer and Computational Sciences 2017 (pp. 721-728). Springer, Singapore.

[9]. Gambhir S, Malik SK, Kumar Y. PSO-ANN based diagnostic model for the early detection of dengue disease. New Horizons in Translational Medicine. 2017 Nov 1;4(1-4):1-8.

[10]. Rao VS, Kumar MN. A new intelligence-based approach for computer-aided diagnosis of dengue fever. IEEE transactions on information technology in biomedicine. 2011 Oct 17;16(1):112-8.

[11]. Murphy J. An overview of convolutional neural network architectures for deep learning. Microway Inc. 2016.

Cite this article as: Mustafa M. et al. Hybrid Convolutional Neural Network with PSO Based Severe Dengue Prognosis Method in Human Genome Data. J. Comput. Sci. Intell. Technol. 2o2o; 1(1): 22-28. @. JCSIT, MNAA PUB WORLD, 2020. 